Article

\title{
Receptive Openness to a Message and Its Dative-Materialist Origin of Time
}

\section{Koichiro Matsuno}

Nagaoka University of Technology, Nagaoka 940-2188, Japan; E-Mail: CXQ02365@nifty.com; Fax: +81429578870

Received: 31 May 2011; in revised form: 20 June 2011 / Accepted: 22 June 2011 /

Published: 1 July 2011

\begin{abstract}
Information precipitates the flow of time from scratch. Information as a noun equivalent of the transitive verb "inform" stands out in the contrast between a direct and an indirect object of the verb, that is to say, between the messenger of a message and its dative. The root of the contrast is sought in the occurrence of the flow of time in the sense that the flow requires both the invariant reference and the dative being subject to something flowing through against the reference. Empirical evidence of the contrast is found in the class identity kept by a molecular aggregate that can constantly exchange the constituent molecular subunits with those of a similar kind available in the neighborhood. The exchange of the subunits derives from the action of pulling-in, originating from the inside of the body holding the class identity. The action of pulling-in that underlies the synthesis of the flow of time empirically in a bottom-up manner originates in the constant update of the present perfect tense in the present progressive tense. The material aggregate preserving the class identity at the cost of the vicissitudes of the constituent individual subunits serves as the dative of information. The unfathomable depth of information is associated with the immense multitude of the messengers in their kinds toward the likely datives having the capacity of receiving them. The bottom line is that being informed is materially being receptive to a flow of substrate, so the information is being embodied by the receptor.
\end{abstract}

Keywords: class identity; clock; dative; genus; individual identity; life; tense; time 


\section{Introduction}

Apart from the difficult nature of answering the question of what is information analytically, it is practically quite pervasive for us to freely refer to the term information both in the scientific discourses and in everyday life, not to mention in philosophy necessarily. Nonetheless, the foundational issue of information still remains equivocal, evasive and unsettled because of the immensely multifarious implications of information itself [1]. The multifarious aspects can readily be visualized as consulting with extreme cases.

For instance, when an aspiring author who had recently published a book sent a simple message "?" as implicitly asking "How well could you sell its copies of mine?" to the publisher, it could happen that the response the publisher sent back to the author might be also another simple one "!" as implying "Very well!" If this information exchange in terms of a very brief message happens to be meaningful to both the parties as being implied, the necessary requirement must be the capacity of receiving messages in a meaningful manner by each participating party irrespectively of whatever may be meant by being meaningful. Another simple example of this sort is bacterial chemotaxis. When bacteria Bacillus subtilis sense and are informed of the direction along which the concentration of repellents such as inorganic salts increases, they tend to avoid the moving in parallel with that direction by tumbling their own bodies repeatedly [2,3]. What underlies bacterial chemotaxis is the receptive capacity of the unicellular organisms themselves as taking the incremental increase of the repellents as the message to be unwelcome, while taking the increase of the attractants such as glucose as the message to be welcome.

These two extreme cases reveal that the term information is a noun equivalent of the transitive verb "inform" like the gerund "informing" derived from it. What is more, information takes the participation of two objects, a direct and an indirect one, for granted as revealed in the instance of informing something of something else. The direct object is a medium to be conveyed in the act of information or more specifically a message to be transferred in the process. And the indirect object is a dative to which the direct object is assigned and given. The dative of the direct object called time, for instance, is just that which records time. The phenomenon of information is thus based upon both the "givenness" of a message as the direct object and the receptive openness toward the givenness on the part of its dative as the indirect object. At issue is how to guarantee the participation of both the givenness of a message and the dative characterized by its receptive openness toward the givenness and on what ground.

\section{Time and Its Dative}

In view of the fact that information is about the movement of relating a direct object to its dative, it would be imperative to admit the participation of what is called time as an indispensable reference to the movement to be referred to as such. Time as a reference to the movement presumes the act of assigning time to the movement. This is nothing other than the act of securing the dative of time. The dative is always about the "aboutness" of the implied direct object.

The present matter of securing the dative of time, however, has posed no serious threat to the current practice of physical sciences attempted so far by accepting the flow of time of exogenous origin as a matter of course as exemplified in classical mechanics. In this regard, Ptolemy's sidereal time is suggestive, which was made explicit in the treatise called the Almagest in the 2nd century A.D. Sidereal 
time remaining unchallenged even until the present day [4], is actually a measure abstracted from the correlated motion that a limited set of stars displayed over the sky. Because of this abstract nature, sidereal time holds no position of being bothered by how its dative could have been abstracted out, any more. Time to Ptolemy was something indexical pointed to with use of something else. Despite that, Newton made time absolute under the articulated supposition of the astronomical equation of time without mentioning its qualification as an abstract idea every time as demonstrated in Philosophiae Naturalis Principia Mathematica.

Newtonian absolute time made after the mold of Ptolemy's sidereal time is a scalar quantity whose sharing among a set of arbitrary moving bodies is the condition for guaranteeing the occurrence of simultaneous correlation among them, while how those moving bodies could have come to share it is left unattended. Once everything happens to appear as riding on the common flow of Newtonian absolute time, synchronous assignment of a common time to every possible dative by a mere stipulation declared from the outside must be the accepted practice there on the employed methodological ground, thus dispensing with the actual act of securing the dative of time from within. In fact, there is no room left for maneuvering the dative of time further once everything has been registered in the finished record, since there is no dative left behind within the record itself. Even any propositional statement made in third person descriptions in the present tense, that has been common in the practice of physical sciences, dispenses with the actual assignment of the dative of time since the whole descriptive domain is declared to share time in the same present tense from the outset. For instance, a set of coupled differential equations of multiple variables framed in the present tense, that is applicable to any present tense, is a simultaneous expression of how these variables are related with each other in a synchronized manner. Nonetheless, this synchronous assignment declared by a sort of decree on the methodological ground alone is not good enough to appreciate and substitute for the actual occurrence of the dative of time. The underlying question is on the concrete scheme of making time flow and assigning the flowing time to its dative, while there is of course no point in worrying about the dative of time in the present tense in general and in hindsight in particular.

Securing the dative of time requires the receptive openness being susceptible to the act of timing being made at the moment of now. The act of timing implied by a transitive verb is grounded upon the occurrence of the now marked by distinguishing the "before" from "after" as referring to the distinction separating between the present progressive and the present perfect tense, at the least. The act of timing is in the happenstance of the present progressive tense punctuated by the occurrence of the present perfect tense. That cannot be covered by the present tense alone. Although physical sciences attempted so far have concentrated exclusively on time in the present tense because of the methodological dismissal of the actual act of securing the dative of time, the prevalence of the present tense could not be tenable any more once this dismissal is duly lifted.

Once the tense of time receives due attention which it deserves, one may expect a likelihood of facing the issue of securing the dative of time head-on while coping with material bodies in movement. When a message, whatever it may be, is given to the dative, the distinction between the act of receiving the message in progress while not yet completed and the receipt already completed would be the most basic empirical attribute to the dative. The distinction to be made in reference to the present moment of the now is within the difference of the tenses of time applied to the dative. The forerunners on this track of addressing the tenses of time in dynamics in a serious manner are philosophers. 
The now as the time to distinguish the present progressive from the present perfect tense, no matter whichever now may be referred to, is of the same quality in exercising the capacity of making such distinctions, while the concrete content of each now could be of the different quality as depending upon how the distinction could be made every time. Aristotle recognized that every now is the same in one respect while any one of it differs from any other of it in other respects (Physics Book IV, 219b). The present ambivalent situation may seem to jeopardize the identity of the now as a noun equivalent of a transitive verb presiding over the flow of time. While the now as a subject must be an identity, it accepts different attributes which might be contradictory with each other in some cases. One diagnosis Aristotle came up with for the task of securing the identity of the now as a legitimate noun accepting a definite set of attributes is that the disparity between the present progressive and the present perfect tense in their implications could eventually vanish in the light of telos or entelecheia to be addressed in the present tense. This diagnosis is, however, not free from its own fault. It has lost the persuasive power as facing Galilei's free-fall experiment of material bodies as demonstrating the irrelevance of the notion of telos. The fact is that despite Aristotle's failure in providing it with a satisfactory answer, the original question on the likelihood of addressing the disparity between the present progressive and the present perfect tense in the present tense remains legitimate and yet to be settled.

The stalemate has persisted even up until the present day. In particular, as late as at the turn of the 20th century, McTaggart made a remark on the intrinsic difficulty in accommodating the issue of addressing the different tenses of time appropriately to legible discourses made in the present tense as revealed in the following quotation:

"What we have done is this - to meet the difficulty that my writing of this article has the characteristics of past, present and future, we say that it is present, has been future, and will be past. But "has been" is only distinguished from "is" by being existence in the past and not in the present, and "will be" is only distinguished from both by being existence in the future. Thus our statement comes to this - that the event in question is present in the present, future in the past, past in the future. And it is clear that there is a vicious circle if we endeavour to assign the characteristics of present, future and past by the criterion of the characteristics of present, past and future [5]".

This is an echo heard from Augustine's original remark "For if there are times past and future, I desire to know where they are. But if as yet I do not succeed, I still know, wherever they are, that they are not there as future or past, but as present. For if there also they be future, they are not as yet there; if even there they be past, they are no longer there. Wheresoever, therefore, they are, whatsoever they are, they are only so as present." (Confessions Book XI, Chapter XVIII, translated by J. G. Pilkington). In essence, the act of an experience is unique to the present or today, while its anticipation is about the future or tomorrow and its memory is about the past or yesterday. Then, the statement like that yesterday's tomorrow is today would come to yield an incomprehensible statement such that the memory of anticipation gives rise to an experience in its implication. Qualities of the past, present and future are different, and assimilating any one of the qualities to any other would be unlikely. As facing this contradictory situation, McTaggart charged time to be qualified further in terms of the three tenses of the past, present and future as being unreal.

Most indicative of the difficulty with addressing the now, that is to say, the disparity between the present progressive and the present perfect tense, in the present tense is to directly face the inescapable 
problem of how the now as a legitimate noun could maintain its identity. In this regard, McTaggart's dismissal of the now in succession as being unreal, though sound and legitimate in its own light, is limited only to the case that the content of each now is assimilated to that of a concrete event at each instantaneous moment. There is however no prior guarantee for limiting each now to each instantaneous moment while keeping its identity as a legitimate noun. This observation comes to suggest the possibility that it might be more appropriate to refer to the flow of time of endogenous origin as a candidate of keeping its own identity from within, rather than to refer to each instantaneous now in succession.

The flow of time of endogenous origin is a legitimate subject matter which Heidegger [6] addressed in a serious manner in the framework of the science of Being rather than in the philosophy of ontology in the traditional sense. What is unique to the flow of time is that by passing away constantly, time remains as time. Time does not disappear even if it constantly passes away at the same time. If the present proposition on the time-flow, though which may seem self-contradictory in its first look, is entertained within the accepted scientific practice, its likelihood would have to be examined against the relevant empirical facts. What Heidegger called our attention to as following Augustine's lead is to paraphrase the Aristotelian proposition stating that the now in one sense is the same but not the same in another, into the empirically testable form stating that time remains as time on one hand while passing away constantly on the other. Once the time-flow happens to be seen as being self-generative, it would necessarily come up with the dative of time as the supporting carrier of the flow. Prerequisite to the occurrence of the dative of time other than the physicist must be the presence of something that can maintain its own identity while processing something else that can constantly pass away. What remains to be seen is how to test it empirically or, if ever possible, experimentally.

\section{The Flow of Time and its Identity}

The dative of time conceived in the theoretical framework of classical mechanics as a prototype of dynamics practiced in physical sciences at large is exceptional. As equating the flow of time to the correlated movement of the stellar configuration, which the astronomer can read and identify over the sky, as summarized in the form of the astronomical equation of time, Newton then provided us in theory with the equation of motion equating the displacement of the stellar configuration in time to the instantaneous property of its configuration in space. Being responsible for specifying and reading the identity of the flow of time in this scheme is only the astronomer who maintains the receptive openness to the givenness of time or timing. This situation limiting the role of the dative of time only to the physicist may remain unchanged even in quantum mechanics and in relativity if it is conceived of exclusively in terms of the equation of motion such as the Schrödinger equation of motion of the wave function.

However, the acclaimed monopoly of the dative of time by the physicist is not self-evident since equating the identity of the flow of time to the correlated movement of the configuration of the material bodies in space is done only in theory there. In contrast, each one of us carrying a wrist watch is confident in reading the watch in a manner to be synchronized with other clocks and watches of a similar nature even if frequent resetting and adjustments are required of them. The process of synchronization on the part of each one of us goes beyond the scope of each watch whose mechanical movement is definitely in accord with what mechanics specifies as perceived by the physicist [7]. The synchronization mediated by the 
frequent intervention of resetting and adjustments in which each one of us participates is the movement in the progress tense frequently punctuated by the interruptions marked by the perfect tense, whereas the astronomical equation of time assumes neither resetting nor adjustments.

In essence, the equation of time entertained in the standard practice of physical sciences takes all of the movable bodies to be parts of a huge clock conceivable only in theory. The identity of the flow of time common to each one of us, on the other hand, is not in the equation of time to the correlated movement of the mechanical parts which each watch incorporates into itself, but within the likelihood of the act of synchronization among those clocks and watches of a similar nature.

Synchronization of the clocks of concrete material makeup with no reference to the astronomical equation of time in theory may make it inevitable to punctuate the progressive tense by the perfect tense even in the absence of the external readers like us provided that these clocks are allowed to interact with each other. What has been specific to the astronomical equation of time is that the identity of the flow of time is substantiated by the identity of the supporting individual material bodies such as a constellation of remote stars. Despite that, if the identity of the flow of time happens to be maintained even without being accompanied by the identity of the supporting individual material bodies, some scheme of implementing the flow of time to be synchronized must be in place. Even each one of us can be confident in securing the identity of the flow of time without being supported by the material identity of the wrist watch carried by. If it requires a repair, we can easily replace it by a new functional one while taking the identity of the flow for synchronization intact as a matter of course.

One more example of the correlated movement which can be suggestive to grounding the identity of the flow of time might be circadian clocks of biological origin. Circadian clocks as the biochemical oscillators coordinating and regulating the metabolic and behavioral activities within the 24-h diurnal cycle are everywhere in biological organisms as ranging from the most primitive photosynthetic bacteria called cyanobacteria and fungi, up to plants, insects and animals.

The robustness of these clocks in multicellular organisms might be ascribed to intercellular interactions. However, even unicellular organisms can also maintain very stable rhythms. A remarkable example of the unicellular class is the clock of the cyanobactrium Synechococcus elongatus that has the correlation time of the clock movement even of order of several months [8]. This clock remains quite robust even though the similar clocks of the different cells in the neighborhood hardly interact with one another, as being different from the case of multicellular organisms. Despite that protein synthesis required to sustain the clock is highly stochastic, the clock itself remains highly stable in a manner of being immune to the intrinsic stochasticity of the underlying biochemical reactions.

The core components of the clock of the cyanobacterium S. elongatus are the three proteins KaiA, KaiB and KaiC. Although circadian rhythms had long been thought to be due primarily to RNA-mediated transcription-translation negative feedback, Tomita et al. [9] demonstrated that KaiC phosphorylation maintains a 24-h rhythm in vivo even if the transcription-translation scheme is inhibited with the result of no KaiC synthesis. In addition, Nakajima et al. [10] further demonstrated that this rhythmic phosphorylation as an indication of circadian rhythm can be reconstituted in vitro only in the presence of KaiA, KaiB, KaiC and ATP as a source of phosphate. Here, KaiC exhibits spontaneous autokinase and autophosphorylase activity. KaiA promotes KaiC phosphorylation and inhibits KaiC dephosphorylation through the protein-protein interaction. KaiB inhibits KaiA effect on KaiC. In fact, KaiA and KaiB are recruited to a C-terminal region of KaiC in a phosphorylation-dependent manner [11]. 
These three Kai proteins in the presence of ATP are necessary and sufficient to sustain a robust oscillation of the phosphorylation level of KaiC.

In particular, a KaiC protein is a homohexamer of the monomeric KaiC subunits. In the phosphorylation phase, KaiA promotes KaiC phosphorylation as being associated with KaiC actively and repeatedly. When the levels of phosphorylation of KaiC reach sufficiently high, the KaiC hexamer comes to associate with KaiB and to inactivate KaiA so as to start the dephosphorylation phase. And the phosphorylation phase will start up again once the level of phosphorylation becomes sufficiently low. At this stage, if both phosphorylation and dephosphorylation proceed on the same KaiC hexamer, it would be extremely unlikely to expect the actual occurrence of the rhythmic cycles in a population of the hexamers because of the intrinsically stochastic nature of biochemical events characterizing each hexamer [12]. One promising candidate for implementing the robust rhythmic coordination of phosphorylation and dephosphorylation in the population of the hexamers could be the shuffling of the monomeric KaiC subunits among the hexamers [13], presumably by means of a combination of the allosteric transition of a KaiC hexamer and the monomer shuffling between the hexamers [14,15].

What has been made explicit here is that the KaiC hexamer maintains its class identity as the hexamer while its monomeric subunits constantly come and go as being exchanged during the rhythmic cycle of phosphorylation and dephosphorylation. The identity supporting the flow of time is not upon the identity of the individual material bodies as with the case of the astronomical equation of time, but is upon the class identity of the hexamer to be maintained by the supporting monomeric subunits while the constituent subunits are constantly exchanged. Time remains as time once the class identity of the KaiC hexamer as the dative of time is attended, while it constantly passes away if the individual monomeric KaiC subunits of the hexamer are focused upon. What is unique to the KaiC hexamer is the fact that it remains identical as a class while being variable in processing its individual components.

The experimental bottom line of the disguise of the KaiC hexamer is such that a predecessor hexamer $\mathrm{K}-\mathrm{K}-\mathrm{K}-\mathrm{K}-\mathrm{K}-\mathrm{K}$ is alternated by the successor $\mathrm{K}^{*}-\mathrm{K}-\mathrm{K}-\mathrm{K}-\mathrm{K}-\mathrm{K}$, and then by $\mathrm{K}^{*}-\mathrm{K} *-\mathrm{K}-\mathrm{K}-\mathrm{K}-\mathrm{K}$ and so on, in which $\mathrm{K}$ is a monomeric KaiC subunit unphosphorylated and $\mathrm{K}^{*}$ is the similar subunit phosphorylated in the presence of ATP as the phosphate source. When the hexamer reaches $\mathrm{K}^{*}-\mathrm{K}^{*}-\mathrm{K}^{*}-\mathrm{K}^{*}-\mathrm{K}^{*}-\mathrm{K}^{*}$, it starts dephosphorylation back to $\mathrm{K}-\mathrm{K}-\mathrm{K}-\mathrm{K}-\mathrm{K}-\mathrm{K}$. What is peculiar here is that although the KaiC hexamer does not undergo the monomer shuffling during the phase of dephosphorylation (from $\mathrm{K}^{*}-\mathrm{K}^{*}-\mathrm{K}^{*}-\mathrm{K}^{*}-\mathrm{K}^{*}-\mathrm{K}^{*}$ to $\mathrm{K}-\mathrm{K}-\mathrm{K}-\mathrm{K}-\mathrm{K}-\mathrm{K}$ ), the phosphorylation phase (from $\mathrm{K}-\mathrm{K}-\mathrm{K}-\mathrm{K}-\mathrm{K}-\mathrm{K}$ to $\mathrm{K}^{*}-\mathrm{K}^{*}-\mathrm{K}^{*}-\mathrm{K}^{*}-\mathrm{K}^{*}-\mathrm{K}^{*}$ ) does require the monomer shuffling in the sense that the hexamer recruits the monomers to be phosphorylated from the outside and lets the unphosphorylated ones go out. This has been the experimentally observed fact.

Of course, we can ask what kind of forces would be lurking behind. At issue here is that if we raise such question, we must have some reliable reference upon which the question thus framed may remain legitimate. Then, the tougher question would be what can serve as that reliable reference. One suggestion in this regard might be available from our acceptance of Galilei's inertia in answering the question of whether the inertia is a plain empirical fact under a certain circumstance or a rational consequence from something else. We may then be asked to respond to a harder question of whether the exchange of materials could be irreducibly fundamental in a manner being incommensurable with Galilei's inertia because of the decisive differences in the circumstantial conditions. What has been 
suggested here is the likelihood of the exchange of materials as another irreducible fundamental property of matter exclusively in an empirical sense.

Of course, the KaiC hexamer as the dative of time does not prevent the physicist as another dative of time from modeling the circadian rhythm with use of the presupposed flow of time whose theoretical identity the physicist reads into the configuration of the underlying molecular constellation. In this form of state dynamics, it is the physicist who relates the identity of the flow of time to the identity of the state attributes. State dynamics is peculiar in seeking the identity of the flow of time in the physicist sitting outside who confirms the identity of the state attributes through their measurements. However, the parameter called time as introduced by the physicist within the adopted theoretical scheme of state dynamics is not nomological in the sense that the KaiC hexamer as the dative of time can survive as a fact, instead of merely as a theoretical explanation, in the circadian oscillation. The identity of the flow of time can be maintained in reference to the class identity of the KaiC hexamer even in the absence of the physicist. The KaiC hexamer turns out to be the dative of time of itself by exchanging the constituent monomeric KaiC subunits, while modeling the circadian rhythm with use of the flow of time lets the physicist be the dative of time.

That the class identity of the KaiC hexamer remains stable and robust points to more than what the underlying individual biochemical reactions would imply. It would certainly be inconceivable to expect such robustness exclusively from those individual reactions alone that are highly stochastic. As a matter of fact, the KaiC hexamer is maintained in the flows of the monomeric KaiC subunits between coming into and going out of the hexamer. What must be observed here is the flow continuity of the monomeric subunits. The actual chemical affinities underlying the maintenance of the class identity of the KaiC hexamer must internally be regulated and differentiated [16] so as to meet the flow continuity of the monomeric subunits. That is the condition of material flow equilibration [17,18], which can function as a dynamic factor for holding the KaiC hexamer robust enough as processing the through-flow of the monomeric KaiC subunits.

Once material flow equilibration is focused upon, it would be straightforward to recognize that the flow disequilibrium between the incoming and the outgoing flow of the monomeric subunits registered in the present perfect tense internally would have to be equilibrated in the subsequent present progressive tense. Otherwise, the condition of flow continuity would be jeopardized in the finished record. Nonetheless, the relationship between the present perfect and the present progressive tense is only sequential and not coincidental. If these two tenses happen to occur in a simultaneous manner, the distinction between the two tenses would have to disappear in contradistinction to the historical fact that each of the two tenses has survived and remained legitimate in its own light. Constant spillover for updating the perfect tense in the progressive tense, which can be assimilated to the prime mover of the flow of time in retrospect, would become inevitable for the sake of material flow equilibration because of the absence of a means for global synchronization all at once. Then, frequent update of the perfect tense in the progressive tense turns out to be a sine qua non for the survival of the class identity of the KaiC hexamer while processing the through-flow of the monomeric KaiC subunits. The flow of time imputed to the survival of the class identity of the KaiC hexamer yields time remaining as time, while the constant exchange of the monomeric KaiC subunits of the hexamer can be associated with time passing away constantly. 
What is unique to the update of the perfect tense in the progressive tense on the part of the KaiC hexamer is that the agent relating the update to the identity of the flow of time is the KaiC hexamer itself, being different from the physicist relating the identity of the state attributes in general or of the stellar configuration in the sky in particular to the flow of time. The identity of the flow of time associated with material flow equilibration is certainly of material origin or, more specifically, of biological origin as far as the circadian rhythms due to Kai proteins are concerned. This observation naturally comes to raise the question of whether the likelihood of the flow of time ascribed to the update of the present perfect tense in the present progressive tense could have been the case protobiologically or even prebiologically.

\section{Citric Acid Cycle in the Absence of Enzymes}

The phenomenon called life is composed of polymers which are also composed of subunits called monomers. Most significant among them are nucleic acids and proteins that are often called biopolymers [19]. The polymers are encapsulated within a membranous boundary whose functions primarily include containment, transport of nutrients and energy transduction. The fundamental process leading to growth of a living system is polymer synthesis such as linking together monomers like amino acids and nucleotides by utilizing energy available within the encapsulated environment. What is unique to nucleic acids is their ability to store and transmit so-called genetic information. In a similar vein, proteins, also called enzymes, have an ability to act as catalysts that can increase the rates of metabolic reactions. The central roles of the genetic and catalytic polymers are found in their incorporation into a cyclic feedback-controlled system, in which information in the genetic polymers is used to direct the synthesis of the catalytic polymers, while the synthesized catalytic polymers then take part in the synthesis of the genetic polymers.

The present brief sketch of a living system in molecular terms now suggests to us a new perspective that a living system is an organized network of chemical reactions, though it may look quite convoluted and complex. One referential framework in this regard is chemical reactions in thermal equilibrium. Reaction kinetics in thermal equilibrium sets the strict condition requiring that every forward reaction is balanced with its reversed reaction. When the four chemical reactants A, B, C, and D are available, for instance, the forward reaction $\mathrm{A}+\mathrm{B} \rightarrow \mathrm{C}+\mathrm{D}$ is balanced by the reversed reaction $\mathrm{A}+\mathrm{B} \leftarrow \mathrm{C}+\mathrm{D}$ in thermal equilibrium. That is the principle of detailed balance, as saying that the population of all of the available chemical reactants remains stationary. No evolution is conceivable within the population of stationary chemical species. There is no chance for the likelihood that new chemical species may come up within the stipulation of detailed balance [20].

However, once the condition of thermal equilibrium is lifted, the stipulation of the population of stationary chemical species would become no more tenable. In fact, our Earth is exposed to the hot energy sources in the form of the solar photons from the sun or the heat from the magma on the periphery of the Earth's core mantle on the one hand and also to extremely cold outer space on the other. Chemical reactions proceeding within the thin surface of Earth can now have an intrinsic potential for sustaining a non-stationary or evolutionary population of reacting chemicals.

One decisive factor for marking the likelihood for an evolutionary population of reacting chemicals is the occurrence of meta-stable products. If one considers the vicinities of hydrothermal vents on the 
seafloor of the ocean of the primitive Earth, chemical reactants riding on hydrothermal circulation of sea-water round the periphery of hot vents would experience huge temperature gradients. The temperature of sea-water in the hot vents could exceed even $350{ }^{\circ} \mathrm{C}$, while the surrounding cold sea-water remains near $0{ }^{\circ} \mathrm{C}$. Chemical reactants in the hot spot can gain the energy to activate various synthetic reactions. At the same time, once the synthesized products are transferred into the surrounding cold seawater, some of them could survive the rapid quenching and could be temporarily stabilized as meta-stable products. Furthermore, by repeating visits to hot spots, the synthesis of meta-stable products could continue rather indefinitely without suffering the strict methodological stipulation of limiting their kinds. That could be evolutionary. In particular, chemical synthesis of meta-stable products as riding on hydrothermal circulation of sea-water round hot vents must have been a major scheme of chemical evolution at the stage prior to the evolutionary onset of photosynthesis upon capturing swift solar photons from the sun.

What is unique to the successive synthesis of meta-stable products is the built-in scheme of letting the succeeding synthesis feed upon the products from the preceding one, though such a scheme could not be conceivable in thermal equilibrium. The sequence of something feeding upon something else requires the participation of a certain mediating vehicle as a resource to be taken in. Once the scheme of feeding upon preceding meta-stable products through some mediating vehicles is set in motion, it can come to gain its pronounced durable identity if a succeeding meta-stable product happens to be of the same kind as a preceding one located somewhere in the upstream. Even if each meta-stable product maintains its identity only temporarily at most, the circulation of the mediating vehicles making the successive production of meta-stable products circular can make itself identifiable externally in a robust manner because of its durable identity. One conspicuous advantage of referring to the material vehicle mediating the successive production of meta-stable products is the likelihood of having reaction cycles. In particular, once a durable reaction cycle happens to appear, it would become addressable in a third person description in the present tense thanks to its empirical robustness without being bothered by having to meet the further requirement of identifying the nature of material agencies involved in an explicit manner, whatever they may be.

One tractable example for examining the empirical possibility of the circulation of material vehicles mediating the running of reaction cycles is the citric acid cycle that can be found in every biological organelle called mitochondria [21]. It synthesizes an energized ATP molecule that serves as an energy currency that is ubiquitous in biology. Basic to the biochemical functioning of the citric acid cycle is this: First, a functional group called an acetyl group $\left(\mathrm{CH}_{3}-\mathrm{CO}-\right)$ released from a pyruvate $\left(\mathrm{CH}_{3}-\mathrm{CO}-\mathrm{COO}-\right)$ as the carbon source and an oxaloacetate (-COO-CO-CH$\left.-\mathrm{COO}-\right)$ as one of the members constituting the cycle are combined to form a citrate (- $\left.\mathrm{COO}-\mathrm{CH}_{2}-\mathrm{COHCOO}-\mathrm{CH}_{2}-\mathrm{COO}-\right)$, in which the symbols within the parentheses are the corresponding chemical formula. For further clarification, when the carbon atoms derived from the supplied acetyl group are denoted by $\mathrm{C}^{\prime}$, the resulting citrate is expressed as (-C'OO- $\left.\mathrm{C}^{\prime} \mathrm{H}_{2}-\mathrm{COHCOO}-\mathrm{CH}_{2}-\mathrm{COO}-\right)$. This first step is a process called acetylation which is to add two carbon atoms from a 2-carbon acetyl group to a 4-carbon oxaloacetate to form a 6-carbon citrate. Second, decarboxylation of a 6-carbon citrate produces a 5-carbon alpha ketoglutarate (-C'OO-C' $\mathrm{H}_{2}-\mathrm{CH}_{2}-\mathrm{CO}-\mathrm{COO}-$ ) via the intervening steps of making a 6-carbon cis-aconitate (-C'OO-C' $\left.\mathrm{H}_{2}-\mathrm{CCOO}=\mathrm{CH}-\mathrm{COO}-\right)$ and a 6-carbon d-isocitrate (-C'OO-C' $\left.\mathrm{H}_{2}-\mathrm{CHCOO}-\mathrm{CHOH}-\mathrm{COO}-\right)$ through dehydration, hydration and dehydrogenation, in which both a 1-carbon carbon dioxide molecule 
and a significant amount of energy are released toward the outside. Third, further decarboxylation of a 5-carbon alpha ketoglutarate produces a 4-carbon succinate (-C'OO-C' $\left.\mathrm{H}_{2}-\mathrm{CH}_{2}-\mathrm{COO}-\right)$ via dehydrogenation, in which a carbon dioxide molecule and a significant amount of energy are also released toward the outside. Finally, a 4-carbon succinate returns to a 4-carbon oxaloacetate (-C'OO-C'O- $\left.\mathrm{CH}_{2}-\mathrm{COO}-\right)$ of the same type as that of the original one via the intervening steps of making a 4-carbon fumarate (-C'OO-C'H=CH-COO-) and a 4-carbon malate (-C'OO-C'HOH-CH$-\mathrm{COO}-$ ) through dehydrogenation, hydration and dehydrogenation.

Then, the oxaloacetate (-C'OO-C'O-CH$-\mathrm{COO}-$ ) can enter into the second round as utilizing the acetyl group $\left(\mathrm{C}^{\prime \prime} \mathrm{H}_{3}-\mathrm{C}\right.$ 'O-) released from a pyruvate $\left(\mathrm{C}^{\prime} \mathrm{H}_{3}-\mathrm{C}\right.$ 'O-O'OO-) for synthesizing another citrate (-C'OO-C' $\mathrm{H}_{2}-\mathrm{C}$ 'OHC'OO- $\mathrm{CH}_{2}-\mathrm{COO}-$ ), in which the carbon atoms derived from the acetyl group for the second round are denoted by $\mathrm{C}^{\prime}$. The end product of the second round is another oxaloacetate (-C'OO-C'O-C' $\mathrm{H}_{2}-\mathrm{COO}-$ ), which can again enter into the third round as utilizing the acetyl group $\left(\mathrm{C}^{*} \mathrm{H}_{3}-\mathrm{C}^{*} \mathrm{O}-\right)$ released from a pyruvate $\left(\mathrm{C}^{*} \mathrm{H}_{3}-\mathrm{C}^{*} \mathrm{O}-\mathrm{C}^{*} \mathrm{OO}-\right)$ for synthesizing one more citrate (-C*OO-C* $\mathrm{H}_{2}-\mathrm{C}^{*} \mathrm{OHC}$ 'OO-C' $\left.\mathrm{H}_{2}-\mathrm{COO}-\right)$, in which the carbon atoms derived from the acetyl group for the third round are denoted by $\mathrm{C}^{*}$. The end product of the third round is one more oxaloacetate (-C*OO-C*O-C' $\left.\mathrm{H}_{2}-\mathrm{C}^{\prime} \mathrm{OO}-\right)$. The cyclic reactions can go round in this manner indefinitely.

Crucial at this point is that while the oxaloacetate synthesized as going round the citric acid cycle once and the one more oxaloacetate located at the starting point certainly belong to the same type, they are not identical with regard to their atomic constituents. The carbon atoms inside the cycle are constantly exchanged by the new ones coming from the outside through acetylation and released toward the outside through decarboxylation. Each carboxylic acid molecule in the cycle is at most meta-stable and cannot hold its individual identity throughout because of the frequent exchange of the constituent carbon atoms with the different individuals of the same type from the outside, that is to say, from a 3-carbon pyruvate supplied externally. Instead, one can make a descriptive access to the citric acid cycle holding its own identity as referring to the circulating carbon atoms of the same type. Although each individual carbon atom in the cycle may serve as the messenger mediating the two agencies of feeding upon and being fed upon the same individual carbon atom, the circulating carbon atoms of the same type would turn out to be a non-causative representation of the messenger accessible in third person descriptions in the present tense since the carbon atoms referred to there are not the same individual atoms. An advantage of referring to the circulating carbon atoms of the same type, instead of each carbon atom holding its own individual identity, is its capability of describing the reaction cycle without explicating the nature of the participating material agencies, whose functioning is inevitable in any case, in an explicit manner.

The present appraisal of types over individual identities has both its merit and demerit. If types are taken to be primary, it would become hard to make a descriptive approach to the emergence of those individuals belonging to a new type that has not been present in advance. Likewise, if individuals are taken to be primary, the descriptive access to them would not always be guaranteed because of the absence of appropriate descriptive types or categories to rely upon for the purpose of pointing to them.

Despite that, carbon atoms as a non-causative representation of the messenger mediating between feeding upon and being fed upon are quite broadly spread out as ranging from tiny ribosome in each biological cell up to the entire biosphere, evolutionarily. At the same time, evolution of the biosphere surrounding the thin surface of Earth consists of processing carbon atoms agentially. Such an agential 
activity is perceivable even in the citric acid cycle operating in every organelle called mitochondria. When the carbon incoming flow into the cycle through acetylation is eventually converted into the carbon outgoing flow from the cycle through decarboxylation, the incoming carbon atoms in the form of an acetyl group ( $\left.\mathrm{CH}_{3}-\mathrm{CO}-\right)$, also seen as a combined form of one carbonyl (-CO-) and one methyl $\left(\mathrm{CH}_{3}-\right)$ group, are required to be transformed into two of carboxyl group (-COO-) until going out of the cycle. Decarboxylation is possible only from a carboxyl group. Since the incoming two carbon atoms belong to one carbonyl and one methyl group, they have to stay inside the cycle for a while until completing the conversion into those to be found in the two of carboxyl group.

In fact, the release of two carbon atoms in the form of two carbon oxide molecules is accomplished not in the first round but in the second and fourth rounds of the reaction cycle after their introduction into the cycle in the form of an acetyl group. If one focuses upon the molecules of oxaloacetate, the substitution of the carbon atoms can be seen to proceed in the sequence of (-COO-CO-CH$-\mathrm{COO}-$ ) $\rightarrow$ (-C'OO-C'O-CH$\left.{ }_{2}-\mathrm{COO}-\right) \rightarrow\left(-C^{\prime}\right.$ 'OO-C'O-C'H $\left.{ }_{2}-\mathrm{COO}-\right) \rightarrow\left(-C^{*} \mathrm{OO}-\mathrm{C} * \mathrm{O}-\mathrm{C}^{\prime \prime} \mathrm{H}_{2}-\mathrm{C}^{\prime} \mathrm{OO}-\right) \rightarrow \rightarrow \rightarrow$ as repeating the cycle. The class identity of the molecule of oxaloacetate can be maintained in the substitution even though the constituent carbon atoms constantly come and go.

This conversion of carbon atoms inside the cycle definitely requires an extremely exquisite, specific and agential control of the rates of various reactions including dehydration, hydration and dehydrogenation in addition to acetylation and decarboxylation. All of these delicate controls over the citric acid cycle housed inside contemporary organelle are accomplished with the aid of a huge armory of enzymes of biological origin. Then, one decisive question would arise with regard to the evolutionary origin of the citric acid cycle. Namely, which must have been prior, either carbon atoms as a non-causative representation of the messenger, mediating the active agencies, or enzymes for actively controlling the rates of various reactions that would form an extremely complex network? If enzymes were prior, this perspective would come to beg a far more difficult question down the road of how could such complex protein molecules called enzymes have emerged in the first place. On the other hand, if carbon atoms as a non-causative representation were prior, one may conceive of a tractable experiment for examining the likelihood of the present perspective. One potential support for this alternative is the observation that the role of an enzyme is to specifically enhance the rate of a particular reaction that could already be possible or available even in the absence of the enzyme.

We have attempted an experiment for running the citric acid cycle in the absence of biological enzymes [22,23]. A rationale for the intended experiment was that if a scheme of the production of the succeeding meta-stable products feeding upon the preceding meta-stable products is implemented in the laboratory, the production sequence could become evolutionary as we have already noted. For this objective, we tried to simulate one type of reaction kinetics riding on hydrothermal circulation of sea-water through the neighborhood of hot vents in the laboratory [24,25]. The experimental citric acid cycle we tried to examine was the reaction scheme of citrate $(6) \rightarrow$ isocitrate $(6) \rightarrow$ alpha ketoglutarate(5) $\rightarrow$ succinate(4) $\rightarrow$ fumarate(4) $\rightarrow$ malate(4) $\rightarrow$ oxaloacetate $(4) \rightarrow$ citrate $(6)$, in which the number in the parenthesis attached to each carboxylic acid is the number of carbon atoms included in that molecule. The source of the carbon flow is pyruvate(3) which supplies the acetyl group(2) right in the middle of the pathway from oxaloacetate(4) to citrate(6) from the outside of the cycle. An abiotic synthesis of pyruvate can be expected from methane thiol as a byproduct of carbonylation reactions at high temperature and pressure sites such as hydrothermal vents in the ocean as using iron monosulphide as a 
catalyst [26]. The synthesis of oxaloacetate may also be conceivable from electrophilic addition of a carbon dioxide(1) into a pyruvate.

We first prepared the reaction solution of $1.0 \mathrm{mM}$ for each of oxaloacetate, citrate, isocitrate, alpha ketoglutarate, succinate, fumarate, and malate, and $10 \mathrm{mM}$ pyruvate as the carbon source. The solution was run through the flow reactor simulating hydrothermal circulation of sea-water from the hot $\left(120^{\circ} \mathrm{C}\right)$ to the cold $\left(0^{\circ} \mathrm{C}\right)$ and back to the hot repeatedly. The flow rate from the hot chamber of volume $15 \mathrm{~mL}$ into the cold chamber of volume $580 \mathrm{~mL}$ was set $10 \mathrm{~mL} / \mathrm{min}$. The reactants passed through a thin nozzle of diameter $0.8 \mathrm{~mm}$ when they entered from the tiny hot chamber to the large cold one. Cycle time of the total volume of fluid $(600 \mathrm{~mL})$ from the hot to the cold and back to the hot was about $60 \mathrm{~min}$, but with stirring the reactants cycled roughly once per minute.

One major result of the experiment over the first 180 min reveals that the concentration of citrate increased from the initial $1.0 \mathrm{mM}$ to $2.15 \mathrm{mM}$ over the first $60 \mathrm{~min}$ [22,23]. The results were reproducible in repeated experiments, especially with regard to the initial buildup of citrate synthesis.

Citrate as a key carboxylic acid molecule constituting the cycle was observed to build up at least initially, while its concentration finally started to decrease because of the experimental stipulation of supplying no additional pyruvate in the process except for those prepared initially. In order to confirm the reaction cycle in operation and the associated carbon through-flow running round the cycle, we next ran the flow reactor with the reaction solution with only the seven member carboxylic acid molecules present with the concentration of $1.0 \mathrm{mM}$ for each molecular species and with no added pyruvate. The results demonstrated only a slight build-up of citrate above the initial concentration of $1.0 \mathrm{mM}$, but remained far less significant compared to the case in the presence of added pyruvate. Pyruvate was found indispensable for the proper operation of the reaction cycle in general and for the carbon through-flow running round the cycle for the buildup of citrate in particular.

Furthermore, we attempted to run the flow reactor in which one of the seven member carboxylic acid molecules was missing in the initially prepared reaction solution. The missing carboxylic acid molecule we focused upon was alpha ketoglutarate, while the concentration of the other six member carboxylic acid molecules was maintained at $1.0 \mathrm{mM}$ for each, along with $10 \mathrm{mM}$ pyruvate. The result revealed no significant build-up of citrate above the initial level of $1.0 \mathrm{mM}$. This observation serves as a testimony to the fact that the build-up of citrate requires an uninterrupted and connected reaction cycle supporting the circulating carbon through-flow round the cycle, which certainly functions as a means of material exchange. All of the seven-member carboxylic acids in the cycle are meta-stable in exchanging their constituent carbon atoms, while the cycle remains quite stable.

Occurrence of the through-flow running round a reaction cycle is regulative in that any incremental increase in the through-flow may decrease the concentration of an arbitrary reactant constituting the cycle when viewed from the upstream toward the downstream, while it can compensate for the decrease when viewed from the downstream toward the upstream. For instance, a slight increase of the carbon through-flow from citrate to isocitrate may decrease the concentration of citrate, while the resulting increase of the through-flow from oxaloacetate to citrate can compensate for the decrease. The regulative capacity latent in the through-flow now tends to make the supporting reaction cycle robust enough against adverse disturbances coming from the outside. The level of the through-flow has to internally be determined and to remain somewhat stable against disturbances of external origin, otherwise there would be no likelihood of having such a robust reaction cycle of internal origin. 
At the same time, if the reaction cycle is really functional in a robust manner, the cycle itself must eventually be able to help synthesizing and recovering the missing member molecule from within. Otherwise, the robustness of the reaction cycle would have to be lost. We certainly observed that the initially missing alpha ketoglutarate could emerge as a member molecule of the cycle at a later stage of the reactor operation. The synthesis of alpha ketoglutarate became identifiable at about $120 \mathrm{~min}$ after the start of the operation and its concentration was identified to be about $0.4 \mu \mathrm{M}$, and the concentration started to increase since then.

These observations, when combined, provide experimental support for the circulating carbon atoms of the same type serving as a non-causative representation of the messenger that can mediate between various agencies. In the experiment, the participating agencies are implemented in the guise of the experimental protocols. Accordingly, naturalization of incorporating material agencies into the prospective citric acid cycle may have been in sight in the actual vicinity of the hydrothermal vents on the seafloor of the primitive ocean. Once naturalization is confirmed, the citric acid cycle would come to have a new implication such that the class property of the individual carbon atoms may uphold the systemic identity of the cycle. The systemic or class identity grounded upon the exchange of material elements of the same types comes into being. This is equivalent to the emergence of some agency being able to perceive the class property of atoms of the same type in a manner being distinguished from taking each atom keeping its own individual identity for granted as practiced in physics.

In the standard practice of physics, it has been common to view each atom of the same type as maintaining its own individual identity even if the resulting individual identities belonging to the same type remain indistinguishable, as echoed in the remark made by Schrödinger on the irrelevance of the exchange of material in physics compared to the cases in biology:

"How does the living organism avoid decay? The obvious answer is: By eating, drinking, breathing and (in the case of plants) assimilating. The technical term is metabolism. The Greek word ( $\mu \varepsilon \tau \alpha \beta \alpha \dot{\alpha} \lambda \varepsilon \varepsilon v)$ means change or exchange. Exchange of what? Originally the underlying idea is, no doubt, exchange of material. (e.g., the German for metabolism is Stoffwechsel.) That the exchange of material should be the essential thing is absurd. Any atom of nitrogen, oxygen, sulphur, etc., is as good as any other of its kind; what could be gained by exchanging them? What an organism feeds upon is negative entropy. Or, to put it less paradoxically, the essential thing in metabolism is that the organism succeeds in freeing itself from all the entropy it cannot help producing while alive [27]”.

However, the physical emphasis on the individual identity of each material element is strictly methodological in its origin. Above all, if one tries to approach the two entities of a macromolecule called an enzyme and each constituent atom at the same time as keeping both identities intact, the task of synthesizing such a macromolecule in a bottom-up manner would become extremely unlikely because of the methodological stipulation of specifying the whereabouts of each constituent atom, both actual and potential. On the other hand, if the synthesis of a macromolecule is integrated into the maintenance of the systemic identity, such as the one specific to the citric acid cycle, without explicit reference to each constituent atom and the associated individual identity, its emergence would become far more likely compared to the cases otherwise with the aid of being anchored at the robust identity of a systemic origin.

Each carbon atom can accommodate into itself at least two different identities. One is the individual identity that is inseparable from the physical body it carries. Another one is the identity preserving the 
class property of a material organization in which each atom takes part, as in the case of the citric acid cycle that is in fact a larger-scale individuality open to the flow of the constituent atomic subunits. If carbon atoms participate in forming the identity of the class property of a material organization which could outlive the individual identity of each constituent atom, the class identity can secure its physical underpinning as competing with the individual identity. Each atom is potentially agential in actualizing its class identity as sensing or measuring the milieu in the immediate neighborhood. Although physics has been extremely competent in specifying the individual identity of each material element, the class property has been appreciated so far exclusively through indistinguishability. The class identity grounded upon indistinguishability, however, is quite limited and meager in its implication empirically.

Once the methodological stipulation of overly emphasizing the individual identity of each atom and molecule is lifted, physics would turn out quite at home with the exchange of material or metabolism despite Schrödinger's observation to the contrary, even at the stage prior to the evolutionary emergence of membranous cellular structures processing the exchange of materials between their inside and outside. A similar short-cut could also be expected for the evolutionary onset of self-replicating molecules such as RNA. If the emergence of self-replicating molecules could be based entirely upon chance events without being helped by any physical vehicle of supporting the once-emerged self-replication, it would have been highly likely to be destroyed as facing hostile disturbances common on the primitive Earth. On the other hand, if the emergence of self-replicating molecules would further be assisted by the enduring systemic identity anchored in the class property of atoms and molecules to be exchanged, the likelihood of sustaining the once-emerged scheme of self-replication could have been enhanced enormously.

\section{Time from Class Identities}

According to the standard practice accepted in physical sciences, the flow of time is relative to the correlated motion of irreducible individual material bodies, though Newton called it absolute because of his unique reference to celestial bodies that are not mundanely Earthbound. The role of the physicist who functions as the dative of time is in the capacity of measuring the correlated motion. Time is assigned to the physicist through the act of measurement of the correlated motion. This observation may raise the likelihood such that time could also be assigned to a material body if it can have the capacity of measurement inside the configuration of material bodies. That is internal measurement $[18,28]$, implying that measurement is ubiquitous on the material basis and is not necessarily monopolized by the physicist.

Measurement makes a distinction between before and after its own act more than anything else. This distinction, though totally foreign to classical celestial mechanics per se, can serve as a harbinger for the genesis of the flow of time on the material basis even without the intervention of the physicist. In addition, internal measurement is required to make explicit something remaining invariant while precipitating the flow of something else for the sake of the flow of time to be registered in the record as such on the material basis, also. This requirement is in parallel with that it is the physicist who comes up with the flow of time from the repetition of the correlated motion in an invariably cyclic manner. However, the association of the repetition of the cyclic motion to the flow of time does not apply to 
internal measurement, since the flow of time associated with the repetition of the cycle is the one that the physicist reads into the cycle in advance as referring to no flows of physical origin. Here, it is the physicist who relates the flow of time to the cyclic motion.

On the other hand, however, the flow of time specific to internal measurement must be some flow of empirical origin which the empirical scientist can read as the flow of time only in hindsight. It also requires some invariant reference against which something to flow through can be discerned. Although the read-into flow of time that is a human artifact in any case remains unquestionably legitimate within the record, such an articulation does not survive right on the spot where the record is just in the process of being made. The issue will be empirical, rather than merely being theoretical or philosophical.

What we have seen is a material aggregate preserving its class identity while constantly exchanging the constituent subunits. The flow of the constituent subunits to be exchanged is grounded upon the class identity of the material aggregate to be preserved. This thesis has been derived directly from the empirically observed facts that have further been analyzed experimentally. Each material aggregate holding its class identity while being involved in the constant exchange of the constituent subunits is robust enough in keeping its own identity. That is the robustness of the material aggregate serving as a clock in the sense that it provides a stable invariant reference against which something passing away constantly is identified [29]. This robustness of the class identity can now provide a unique perspective toward the origins of life and beyond.

One relevant issue must be the synchronization of the clocks of material origin in the absence of the physicist who can read into the flow of time in advance from the outside. Since each clock is a robust material aggregate processing constant material through-flow, it can synchronously interact with a similar clock in the neighborhood by exchanging materials. What is unique to the synchronous interaction between the two clocks is that these two implement the mutual interaction while not losing each class identity in the process. Underlying the synchronous interaction is the capacity of modifying each clock so as to be susceptible to the other while keeping the robust class identity of its own. Its consequence is the synchronization between the two clocks without referring to the flow of time that may be read into by the physicist.

Synchronization is a phenomenon of any pair of clocks involved in the exchange of materials between them as keeping each class identity secured in a robust manner. Nonetheless, while it is certainly physical, synchronization upon the exchange of materials has not received due attention which it deserves as a legitimate physical process, so far. One reason behind is that the exchange of materials in focus is internally driven for the activity of feeding upon the outside while experiencing the deficiency from the inside, instead of being forced externally from the outside as readily comprehended within the standard framework of physics. This exchange of materials from within is based upon the receptiveness of the dative pulling in the materials to be exchanged, and cannot properly be addressed in the present tense from the outside. For the materials positioned at the dative in the upstream are constantly being further pulled down into another dative in the downstream. The dative as an indirect object is constantly being converted into the direct object in the sequence of the pulling action, while the present tense is not competent enough by itself to distinguish whether the object is progressively a direct or an indirect one or whether it is being pulling in or is being pulled in. In contrast, the present tense does not change its implication whether it may be framed in the active voice or in the passive one. What is occurring here is the constant update of the present perfect tense in the present progressive tense, which is inaccessible to 
the present tense alone as a matter of principle. Even physics as we now know of it is potentially competent in appreciating the process of updating the perfect tense in the progressive tense in the framework of irreversible thermodynamics as reminding us that thermodynamics practiced strictly in the present tense remains under-complete. The cohesion acting between the perfect and the progressive tense is unique in that each tense pulls in the other for the start-up of the latter, but both cannot coexist since the tenses of a verb are mutually exclusive in their implications due to the incompatible contrast between having been completed and being continuous in progress.

On the other hand, the tradition according to which we have practiced physics so far is first to accept time in the present tense as if the occurrence of the tenses other than the present would be irrelevant to physics and then to figure out the action of pulling in, if any. Needless to say, the pulling action or the attractive interaction of relevance to biology attempted in physics has been the interaction accessible only in the present tense like the electrostatic one including those further modifications by the medium in the immediate neighborhood such as surface tension and van der Waals attractions. However, once the stipulation of limiting to the present tense is lifted, the likelihood of the pulling action upon the dynamic attraction processing the mediation between the different tenses, other than that upon the static attractive interactions restricted only to the present tense, could come up to the surface as a legitimate physical means for implementing the exchange of materials. The action of pulling in, that is agential in feeding upon the outside because of the deficiency experienced from the inside, does not presuppose the phenomenon of life. Rather, the phenomenon of life is based upon the action of pulling in that is also operative in the form of the sustainable feed-forward loop [30] and anticipatory at the same time. Cells are not prerequisite to the origins of life. Rather, the action of pulling in deriving from the material bodies assuming the dative of time is prerequisite to the evolutionary emergence of cells.

Synchronization proceeding between whatever clocks of material origin in the empirical domain is about the material process of precipitating the flow of time to be registered and shared in the present perfect tense in the finished record without presupposing the flow of time as physics has taken it for granted. Even if the flow of time is referred to in the record, there would be no likelihood such that the prior material through-flow for synchronization may refer to the posterior flow of time for its own operation. This is due simply to the fact that the material through-flow mediating the synchronization between any pair of clocks can be associated with the flow of time only in retrospect, while the act of synchronization proceeds in processing the material through-flow and not in following the presupposed flow of time. Consequently, there is no prior guarantee for the likelihood of synchronization when a pair of clocks comes to interact with each other through the exchange of materials between the two. The likelihood for synchronization is totally an empirical issue. If either one of the two clocks happens to lose its robustness upholding the class identity of a material aggregate processing the material through-flow during the interaction with the other, no chance of synchronization between the two could be envisioned with the consequence of no flow of time to be shared in retrospect.

One unique property of synchronization applied to a configuration of clocks is that it can serve as a factor indicating the occurrence of a self-organization proceeding in the configuration-space in the sense that the flow of time would come to be shared unanimously all over the space in the course of events. In contrast, physics to be practiced in the strict mechanistic scheme is peculiar in admitting the completion of such a self-organization from the outset. This completion is upon the methodological ground of the flow of time to be shared unanimously in the form of the equation of motion as demonstrated in a 
parametric representation of the self-organization completed in terms of time. Despite that, physics has still left room for self-organization to some extent.

One likely candidate for self-organization is found in thermodynamics, which remains under-complete in the sense that all of the thermodynamic variables cannot be determined within the given theoretical framework alone. For instance, although it can give a definite relationship among the volume, the pressure and the temperature of a gas as framed in the Boyle-Charles' law in a third person description in the present tense, thermodynamics does not determine each value of the three variables in a definitive manner. There remains some room for self-organization or for the action of pulling in even within the framework of thermodynamics in that how the synchronization of the clocks embodied in the robust material aggregates processing the through-flows available there could proceed has been left unattended externally. Although it remains under-complete when practiced in the present tense, thermodynamics still remains open to how it could be practiced as facing the update of the perfect tense in the progressive tense. Thermodynamics is unique in implicitly accepting the dative of time other than that being applicable only to the present tense. In fact, thermodynamics is potentially capable of addressing how the clocks available there could be synchronized among themselves. The actual act of self-organization through synchronization comes to be addressable in the update of the present perfect tense in the present progressive tense, rather than in the present tense alone.

One more likely candidate for the self-organization conceivable within physics is available from quantum mechanics, which also remains under-complete unless the act of measurement is supplemented. Since measurement makes a quantum distinction between before and after its own act, it provides a scheme of punctuating the progressive tense by the perfect tense and updating the perfect tense in the progressive tense. The dative of time of material origin can be internalized in quantum mechanics unless it is expelled merely on the methodological ground. Measurement is intrinsically nonlinear in the practice of quantum mechanics in synchronizing both the present perfect and the present progressive tense in its own act. That is the punctuation of the progressive tense by the perfect tense. Nonlinearity latent in practicing quantum mechanics certainly witnesses room for cultivating the scheme of self-organization further upon the built-in act of synchronization.

Unless it is taken as a criterion of the self-organization completed, as revealed from even within physics in a comprehensible manner, the flow of time for self-organization as concretized in the form of synchronization is exclusively empirical. The empirical nature of the flow of time in hindsight is found within its association with the flow of something physical. Time-experiencing is associated with experiencing the material through-flow on the part of a material aggregate holding its class identity. That is to say that the material aggregate holding its class identity can serve as the dative mediating between the flow of something physical on the scene and the flow of time in retrospect. What is advantageous to the dative is that it can distinguish the message of a physical nature to be represented as the flow of time from the flow itself. The dative can maintain the capacity of translating the message into the actual implied object that may turn out the direct object having an agential capacity as a subject in the subsequent round. This translation of the message into the implied object underlies the ubiquity of the flow of time in the empirical world.

If one wants to apply the notion of time to the empirical world at large, there could be at least two options. One is to presuppose the ubiquity of the flow of time in a transcendental manner and to let every other thing move on that flow read into by the physicist. Although this option has widely been accepted 
in physics, it cannot get rid of its built-in transcendental stipulation. There is no likelihood of expecting the agential capacity in the form of the action of pulling in within the framework of the flow of time read into by the physicist. One more option is to limit the application of the flow of time only to those datives that can come to synchronize with each other as maneuvering a wide variety of material means to be represented effectively as the flow of time instead of directly maneuvering the flow itself.

Once the dative of the flow of time receives due attention, it would become feasible, for instance, to synchronize the quartz watch as a human invention with the cyanobacterial circadian clock of a natural kind while handling only the material substrates to be represented as the flow of time, without referring to the flow directly. Although the flow of time is a highly anthropocentric notion, the dative of a natural kind precipitating the flow of time in hindsight can be naturalized in the sense that it can have the capacity for the task of synchronizing with those other datives of a similar kind, including humans too, that are available in the neighborhood.

The notion of the flow of time is peculiar in presuming the act of synchronization that has already been completed within whatever system to which the flow is applied. In contrast, an advantage of focusing upon the dative of the flow of time is in the appraisal of the bottom-up process of an empirical nature for approaching the synchronization from within. Focusing upon the dative mediating the flow of something physical and the flow of time in hindsight relieves us from committing solely to the top-down imposition of synchronization in a transcendental manner. The present relief from the top-down stipulation is more than merely on the issue of the flow of time. It can also apply to the perennially controversial issue of information.

A key agenda surrounding the issue of information is that whatever information may imply, its dative called the recipient of a message transforms what is conveyed by the physical messenger into the actual object to further be processed. The dative is required to equip itself with the receptive openness to a likely messenger to come in. The receptive openness thus resides in the capacity of passive integration on its own that cannot be stifled by the top-down stipulation of mechanistic control from the outside. One likely primitive case of the receptive openness is evident in the dynamic chemical affinity exhibited by carboxylic acid molecules feeding upon the upstream of the carbon flow in the citric acid cycle. The messenger to carboxylic acids in the citric acid cycle as functioning as the datives is the carbon atoms. As a matter fact, the multitude of the messengers to be involved remains unfathomable in advance. Fixation of the carbon atoms as the messenger is due simply to the historical uniqueness of the occurrence of the citric acid cycle.

Another more convoluted case is the action potential reaching toward the motor neuron terminal and causing a calcium ion influx through the voltage-gated channels. The calcium-ion influx causes vesicles containing the neurotransmitter acetylcholine to fuse with the plasma membrane and lets the vesicles release acetylcholine out into the extracellular space between the motor neuron terminal and the motor end plate of the muscle cell. The messenger to the datives identified as the vesicles containing the neurotransmitter acetylcholine is the calcium-ions. Then, the activated acetylcholine receptors on the motor end plate serving as the datives to the messenger acetylcholine trigger an action potential to be propagated further down along the muscle fiber. What is significant here is that the extra amount of idled acetylcholine floating in the synaptic cleft region is soon destroyed by acetylcholine esterase and the receptive openness toward the next arrival of the calcium-ion influx is recovered. 
In short, integrating the dichotomy of the messenger and its dative into a synthetic organization provides an information-specific naturalization of constructing the flow of time in a bottom-up manner. Information makes time synthetic. That is to say, information serves as a cohesive factor for precipitating the flow of time from scratch. One irrevocable advantage of the information-specific perspective is to remind us that contrary to the superficial intransitive nature in its outlook, the flow of time does require a transitive verb contrasting the direct with the indirect object for its own operation. Likewise, a proper appraisal of the information-specific perspective is required to get rid of the read-into flow of time of an imposed character that has been common in the standard practice of physics; otherwise the synthetic capacity of information would be jeopardized. We can thus see that both the proper flow of time and information are parallel in emphasizing the synthetic capacity as grounding each of them upon the activity of holding the class identity of a material aggregate as processing the flow of the constituent material subunits from within by means of the action of pulling in, while the analytical features are totally different between the two.

\section{Concluding Remarks}

It must be a queer coincidence that both issues of the flow of time and of information touch upon the linguistic condition of the presence of a transitive verb requiring both a direct and an indirect object. In fact, the occurrence of the dative as an indirect object to a transitive verb is inevitable to the empirical world once the priority of sense perception is legitimately appreciated. Sense perception on the part of the dative takes some physical stimulus as a sign for doing something else as practiced in semiotics in general or in biosemiotics in particular [31]. What is more, although the transitive verb presupposes the contrast between the subject and the dative, there should be no transcendental subject presiding over all of the other datives since the tentative subject of a transitive verb to be met in the empirical world has been the former dative one step or more earlier in the convoluted network spanned by a wide variety of transitive verbs.

Letting everything be the dative in one form or another, however, does not imply that the movement paraphrased in transitive verbs could eventually reduce to the motion ascribed to an intransitive verb as implicated by celestial mechanics. Quite the contrary, each dative can locally be active and agential on its own in sorting out the messenger to be received and accepted as such although the messengers remain inert in themselves. The dative is thus only reactively active or passively synthetic.

The present reactive nature of activity with the dative must have become most acute on the verge of the origins of life at least on the planet Earth. In particular, a decisive factor for approaching the distinction between non-life and life is the class identity of the dative that can outlive the individual identity of the messenger to participate in the proper functioning of the former. Conceiving an evolutionary scenario on crossing the gap between non-life and life as following the flow of time read into by the physicist, though which may run quite smoothly as a plausible discourse, is not legitimate for uncovering the underlying evolutionary dynamics because of the incompatible nature of the dative of time employed there. An evolutionary scenario riding on the flow of time of exogenous origin cannot address the actual endogenous update of the present perfect tense in the present progressive tense. The temporal sequence of events found either in whatever evolutionary scenario including the adaptationist 
one or in the finished record, though legitimate in its own light, can neither be about nor substitute for the actual causal nexus underlying the constant update of the perfect tense in the progressive tense.

The distinction between non-life and life can be reduced to the distinction between the class identity of the dative of time and the individual identity of the messenger to be exchanged, the latter distinction of which can be approachable empirically or even experimentally. One can thus find here a clue for addressing the issue of the origins of life without using the notion of life as an analytical term that would otherwise require a formidable task of framing the precise definition of the term for its own sake. The situation with the notion of information is similar. Although it is referable in a synthetic sense, information meets insurmountable difficulties in conceiving it as an analytical tool because of its intrinsically synthetic implication.

In fact, the three issues of the flow of time, the phenomenon of life and the notion of information share one thing in common. That is the priority of the class identity over the individual identity of the subunit in the sense that the class identity is kept at the cost of the vicissitudes of the individual identities of the constituent subunits through the exchange with the others of a similar kind from the outside. The class identity is held upon the action of pulling-in that is internal to the body constituting the class itself. This priority of the class identity exhibits a marked contrast to the Aristotelian dictum saying that being is not a genus (Metaphysics Book III, 998b) with the implicit implication of the universality of being. However, whether or not the class identity is prior, is a matter to be examined empirically rather than being merely a matter of theoretical or philosophical thesis. Even if it may look one step backward, the appraisal of the class identity over the individual identity might be the price to pay to make two steps forward.

\section{References}

1. Capurro, R.; Fleissner, P.; Hofkirchner, W. Is a unified theory of Information feasible? A trialogue. World Futures 1997, 49, 213-234.

2. Ordal, G.W. Recognition sites for chemotactic repellents of bacillus subtilis. J. Bacteriol. 1976, 126, 72-79.

3. Eisenbach, M.; Constantinou, C.; Aloni, H.; Shinitzky, M. Repellents for Escherichia coli operate neither by changing membrane fluidity nor by being sensed by periplasmic receptors during chemotaxis. J. Bacteriol. 1990, 172, 5218-5224.

4. Barbour, J. The nature of time, 2009. Available online: http://arxiv.org/abs/0903.3489 (accessed on 22 June 2011).

5. McTaggart, J.E. The unreality of time. Mind: A Q. Rev. Psychol. Philos. 1908, 17, 456-473.

6. Heidegger, M. Zeit und sein. In Zur Sache des Denkens; 5th ed.; Niemeyer: Tübingen, Germany, 1969; pp. 1-25.

7. Matsuno, K. Dynamics of time and information in dynamic time. BioSystems 1998, 46, 57-71.

8. Mihalcescu, I.; Hsing, W.; Leibler, S. Resilient circadian oscillator revealed in individual cyanobacteria. Nature 2004, 430, 81-85.

9. Tomita, J.; Nakajima, M.; Kondo, T.; Iwasaki, H. No transcription-translation feedback in circadian rhythm of KaiC phosphorylation. Science 2005, 307, 251-254. 
10. Nakajima, M.; Imai, K.; Ito, H.; Nishiwaki, T.; Murayama, Y.; Iwasaki, H.; Oyama, T.; Kondo, T. Reconstruction of circadian oscillation of cyanobacterial KaiC phosphorylation in vitro. Science 2005, 308, 414-415.

11. Akiyama, S.; Nohara, A.; Ito, K.; Maeda, Y. Assembly and disassembly dynamics of cyanobacterial periodosome. Mol. Cell 2008, 29, 703-716.

12. Emberly, E.; Wingreen, N.S. Hourglass model for a protein-based circadian oscillator. Phys. Rev. Lett. 2006, 96, 038303-038306.

13. Kageyama, H.; Nishiwaki, T.; Nakajima, M.; Iwasaki, H.; Oyama, T.; Kondo, T. Cyanobacterial circadian pacemaker: Kai protein complex dynamics in the KaiC phosphorylation cycle in vitro. Mol. Cell 2006, 23, 161-171.

14. Eguchi, K.; Yoda, M.; Terada, T.P.; Sasai, M. Mechanism of robust oscillation of KaiC phosphorylation in vitro. Biophys. J. 2008, 95, 1773-1784.

15. Nagai, T.; Terada, T.P.; Sasai, M. Synchronization of circadian oscillation of phosphorylation level of KaiC in vitro. Biophys. J. 2010, 98, 2469-2477.

16. Zwicker, D.; Lubensky, D.; ten Wolde, P.R. Robust circadian clocks from coupled protein-modification and transcription-translation cycles. Proc. Natl. Acad. Sci. USA 2010, 107, 22540-22545.

17. Matsuno, K. Open systems and the origin of protoreproductive units. In Beyond Neo-Darwinism; Ho, M.W., Saunders, P.T., Eds.; Academic Press: London, UK, 1984; pp. 61-88.

18. Matsuno, K. Internal Measurement. Protobiology: Physical Basis of Biology; CRC Press: Boca Raton, FL, USA, 1989; Chapter 2.

19. Deamer, D. Special collection of essays: what is life? Astrobiology 2010, 10, 1001-1002.

20. Matsuno, K. Carbon atoms as prime messengers for the origins of life. In Messages and Messengers - Angeletics as an Approach to the Phenomenology of Communication; Capurro, R., Holgate, J., Eds., Wilhelm Fink Verlag, Paderborn, Germany, 2011; pp. 303-325.

21. Morowitz, H.J.; Kostelnik, J.D.; Yang, J.; Cody, G.D. The origin of intermediary metabolism. Proc. Nat. Acad. Sci. USA 2000, 97, 7704-7708.

22. Matsuno, K.; Nemoto, A. Quantum as a heat engine-the physics of intensities unique to the origins of life. Phys. Life Rev. 2005, 2, 227-250.

23. Matsuno, K. Forming and maintaining a heat engine for quantum biology. BioSystems 2006, 85, 23-29.

24. Matsuno, K. A design principle of a flow reactor simulating prebiotic evolution. Viva Origino 1997, 25, 191-204.

25. Imai, E.; Honda, H.; Hatori, K.; Brack, A.; Matsuno, K. Elongation of oligopeptides in a simulated submarine hydrothermal system. Science 1999, 283, 831-833.

26. Cody, G.D.; Boctor, N.Z.; Filley, T.R.; Hazen, R.M.; Scott, J.H.; Yonder, S.H., Jr. Primordial carbonylated iron-sulfur compounds and the synthesis of pyruvate. Science 2000, 289, 1337-1340.

27. Schrödinger, E. Order, Disorder, and Entropy. What is Life?-The Physical Aspect of the Living Cell; Cambridge University Press: Cambridge, UK, 1944; Chapter 6.

28. Matsuno, K. Internalist stance and the physics of information. BioSystems 1996, 38, 111-118.

29. Matsuno, K. The clock and its triadic relationship. Semiotica 1999, 127, 433-452. 
30. Rosen, R. Life Itself: A Comprehensive Inquiry into the Nature, Origin, and Fabrication of Life; Columbia University Press, New York, NY, USA, 1991.

31. Matsuno, K. Framework of space and time from the proto-semiotic perspective. Biosemiotics 2011, 4, 103-118.

(C) 2011 by the authors; licensee MDPI, Basel, Switzerland. This article is an open access article distributed under the terms and conditions of the Creative Commons Attribution license (http://creativecommons.org/licenses/by/3.0/). 